\title{
Molecular tori in AGN: \\ a search using excited states of $\mathrm{OH}$
}

\section{M. Violette Impellizzeri ${ }^{*}$}

Max-Planck-Institut für Radiostronomie, Bonn, Germany

E-mail: violette@mpifr-bonn.mpg.de

\section{Alan L. Roy, Christian Henkel}

Max-Planck-Institut für Radiostronomie, Bonn, Germany

E-mail: aroy@mpifr-bonn.mpg.de chenkel@mpifr-bonn.mpg.de

One of the fundamental concepts in the unified scheme of active galactic nuclei is that both Seyfert 1 and Seyfert 2 galaxies harbour supermassive nuclear engines blocked from direct view by an optically and geometrically thick torus. If the pressure is sufficiently high, the content of the torus should mostly be molecular. Although molecular rings with diameters of a few hundred parsecs are common, the expected small scale tori $(<10 \mathrm{pc})$ have been difficult to detect. Searches for absorption lines of common molecules like $\mathrm{CO}$ and $\mathrm{OH}$ have mostly resulted in non-detections. Before concluding that tori are not molecular, radiative excitation effects, in which coupling to the nonthermal continuum can suppress the opacity in the lowest transitions, deserve some attention and influence our selection of the most favourable transitions to observe. To explore these effects, we modified the search strategy by looking for the higher excited rotational states of $\mathrm{OH}$ and by selecting a sample of 31 Seyfert 2 galaxies which are known to have a high X-ray absorbing column. We present here the results of single dish observations of the transitions at $6031 \mathrm{MHz}$ and $6035 \mathrm{MHz}$, yielding detections in five sources. We also present a spectral line VLBI observation carried out at $13.4 \mathrm{GHz}$ towards the core of Cygnus A, yielding a tentative detection.

The 8th European VLBI Network Symposium

September 26-29, 2006

Toruń, Poland

${ }^{*}$ Speaker. 


\section{Molecular tori in AGN}

Active galactic nuclei (AGN) come in two main types: those with and those without broad optical line emission (type 1 and type 2 AGN, respectively). In the unified scheme of active galactic nuclei, all AGN are intrinsically similar; in type 2 objects our view of the central continuum source and the broad line region (BLR) is blocked by an obscuring material of significant column density [1]. The obscuring material is expected to be an approximately parsec-scale torus of molecular gas, whose structure was predicted by, e.g., Krolik \& Begelman [3]. Despite many efforts to detect the expected molecular absorption or emission in a number of surveys, only in very few cases could molecular absorption be confirmed (e.g. Schmelz et al. 1986, Baan et al. 1992, Staveley-Smith et al. 1992 [П], [5], [6]). The unified scheme accounts for many observed properties, but evidence for the existence of a molecular torus remains somewhat indirect and the structure and extent of the obscuring material are still poorly understood.

As the nearest $(z=0.0565)$ powerful FR II radio galaxy, Cygnus A has been the subject of a number of studies aimed at testing the predictions of the unified scheme. Several lines of evidence suggest that Cygnus A harbours a quasar nucleus hidden from direct view in the visible and soft X-rays emission by a dusty obscuring torus [7]. An X-ray absorbing column of $N_{\mathrm{H}}=(3.75 \pm$ 0.73) $\times 10^{23} \mathrm{~cm}^{-2}$ is consistent with the notion of a buried quasar in Cygnus A [8]. Searches for $18-\mathrm{cm} \mathrm{OH}$ and $6-\mathrm{cm} \mathrm{H}_{2} \mathrm{CO}$ by Conway \& Blanco [9] yielded non-detections at $1 \%$ optical depth [9]. Also, Barvainis \& Antonucci [10] failed to detect CO J=0-1 and CO J=1-2 absorption, challenging the torus model for Cygnus A. The authors suggested three possible solutions. First, it may be that there is no such torus. Second, the sizes of the molecular clouds in the torus may be smaller than the size of the background continuum source. Alternatively, the radio continuum emission from the nucleus may radiatively excite the $\mathrm{CO}$, increasing the excitation temperature of the lower rotational levels and suppressing the absorption optical depths in the lower transitions [2]. Interestingly, Fuente et al. [11] report 118-GHz CO+ absorption, supporting $\mathrm{CO}$ excitation from radiative excitation effects. Given the abundances of $\mathrm{OH}$ predicted in some molecular torus models, the non-detection of $\mathrm{OH}$ absorption is hard to explain unless this, too, is radiatively excited. Radiative excitation effects have been predicted by Black (1997) also for the 18-cm OH transitions [12], suggesting that the rotationally-excited doublets at $6 \mathrm{GHz}$ and $13.4 \mathrm{GHz}$, so far neglected, could be more profitable targets than the 1.6-GHz lines.

\section{Observations}

To explore the effects of radiative excitation we modified the search strategy for molecular absorption, starting with a survey with the 100-metre telescope in Effelsberg to search for $\mathrm{OH}$ in the the higher-order transitions at $6031 \mathrm{MHz}$ and $6035 \mathrm{MHz}$ in 31 type $2 \mathrm{AGN}$ (see Table 1) selected for having a known high X-ray absorbing column $\left(\geq 10^{22} \mathrm{~cm}^{-2}\right)$ and for having $S_{6 \mathrm{GHz}}>50 \mathrm{mJy}$ to ensure sufficient continuum core strength. We included some AGN with polarized broad-line emission, indicating an obscured BLR, and some additional sources with known $\mathrm{OH}$ absorption at $1.6 \mathrm{GHz}$. The observations were carried out between August 2003 and February 2004 with an average integration time of $3 \mathrm{~h}$ per source and $40-\mathrm{MHz}$ bandwidth. Twenty of the stronger sources 


\begin{tabular}{|c|c|c|c|c|c|c|c|c|c|c|c|}
\hline Source & $\begin{array}{c}\text { Redshift } \\
z\end{array}$ & $\begin{array}{l}S_{6 \mathrm{~cm}} \\
\mathrm{mJy}\end{array}$ & $\begin{array}{l}N_{\mathrm{H}} \mathrm{X} \text {-ray } \\
\mathrm{cm}^{-2}\end{array}$ & $\begin{array}{l}\text { Int. time } \\
\min \end{array}$ & Det & Source & Redshift & $S_{6 \mathrm{~cm}}$ & $N_{\mathrm{H}} \mathrm{X}$-ray & Int. time & Det \\
\hline Hydra A & 0.055 & 154 & - & - & & NGC 5135 & 0.014 & 598 & $>10^{24}$ & 120 & - \\
\hline Cygnus A & 0.056 & 1400 & $10^{23.5}$ & - & - & NGC 5506 & 0.006 & 160 & $3.4 \times 10^{23}$ & 230 & - \\
\hline NGC 1052 & 0.005 & 1500 & $>10^{22}$ & - & - & NGC 5793 & 0.011 & 96 & & 230 & abs \\
\hline NGC 1068 & 0.004 & 1090 & $>10^{25}$ & - & - & NGC 6240 & 0.024 & 131 & $2.2 \times 10^{25}$ & 103 & - \\
\hline NGC 1167 & 0.002 & 243 & $<2 \times 10^{23}$ & 154 & - & NGC 7130 & 0.016 & 62 & $>10^{24}$ & 180 & - \\
\hline NGC 1275 & 0.017 & 16623 & $1.5 \times 10^{23}$ & - & - & NGC 7674 . & 0.029 & 66.5 & $>10^{24}$ & 205 & - \\
\hline NGC 1365 & 0.006 & 191 & $2 \times 10^{24}$ & 180 & - & Mrk 3 & 0.014 & 361 & $1.1 \times 10^{25}$ & 205 & - \\
\hline NGC 1808 & 0.003 & 207 & $3 \times 10^{23}$ & 180 & - & Mrk 231 & 0.042 & 414 & - & $\dagger$ & abs \\
\hline NGC 2110 & 0.007 & 175 & $2.9 \times 10^{23}$ & 180 & - & Mrk 273 & 0.037 & 103 & - & $\dagger$ & abs \\
\hline NGC 2639 & 0.040 & 54.5 & - & 154 & - & Mrk 348 & 0.015 & 254 & $10^{24}$ & 211 & - \\
\hline NGC 2992 & 0.008 & 77 & $6.9 \times 10^{22}$ & 185 & - & Mrk 463 & 0.050 & 100 & $1.6 \times 10^{24}$ & 180 & - \\
\hline NGC 3079 & 0.004 & 145 & $1.6 \times 10^{23}$ & 65 & abs & Mrk 1210 & 0.014 & 45 & $10^{25}$ & 180 & - \\
\hline NGC 4151 & 0.003 & 125 & $2.2 \times 10^{22}$ & 255 & - & Mrk 1073 & 0.024 & 44 & - & 358 & - \\
\hline NGC 4261 & 0.007 & 80 & - & 180 & abs & F 01475-0740 & 0.177 & 127 & - & 103 & - \\
\hline NGC 4388 & 0.008 & 76 & $4.2 \times 10^{24}$ & 205 & - & IRAS $05414+5840$ & 0.015 & 55 & - & 180 & - \\
\hline & & & - & & & IRAS $1345+1232$ & 0.121 & 2160 & - & 180 & - \\
\hline
\end{tabular}

Table 1: List of sources observed at $6 \mathrm{GHz}$ with the $100-\mathrm{m}$ telescope in Effelsberg. $\dagger$ sources observed by Henkel et al. (priv. comm.)

were followed up in the 4.7-GHz transitions with Effelsberg telescope between June 2004 and August 2004.

Four of our top candidates: Cygnus A, Hydra A, NGC 1052 and NGC 1275, were too strong for single-dish spectroscopy, and so demanding unachievably high spectral dynamic range and for two of these we thus proposed an interferometric study. We looked for excited $\mathrm{OH}$ at $13.4 \mathrm{GHz}$ in Cygnus A and NGC 1052 with the VLBA, omitting Hydra A since its core was too weak at 13.4 GHz. The observations were made with two IFs of $16-\mathrm{MHz}$ bandwidth (corresponding to $357 \mathrm{~km} / \mathrm{s}$ per IF). The IFs were centred at $13.434 \mathrm{GHz}$ and $13.441 \mathrm{GHz}$, respectively, with 256 channels per IF, yielding a spectral line resolution of $1.4 \mathrm{~km} / \mathrm{s}$ per channel. The data were calibrated using standard phase and amplitude calibration procedure, using 2005+403 and 2013+370 for bandpass calibration of Cygnus A, and 0423-01 and 3C84 for NGC 1052.
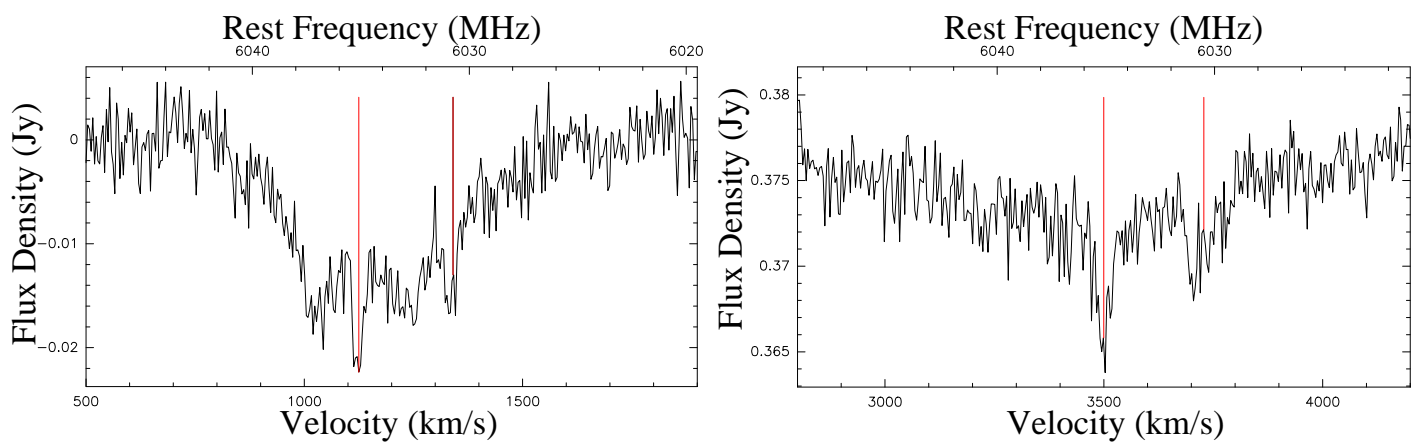

Figure 1: Absorption spectra of NGC 3079 (left) and NGC 5793 (right). The red lines mark the position of the two main lines at $6031 \mathrm{MHz}$ and $6035 \mathrm{MHz}$ at the systemic velocity. The velocity scales refer to the 6035-MHz line. 


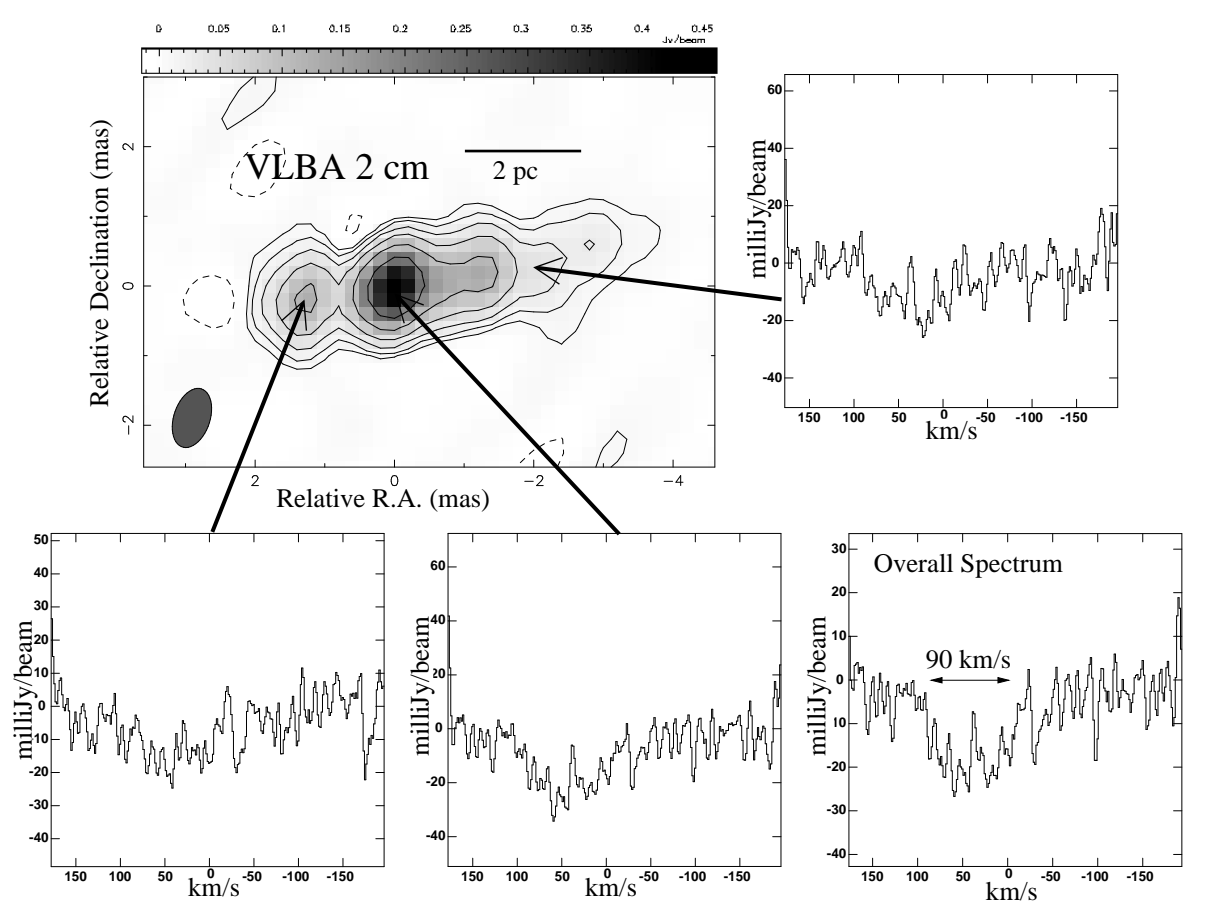

Figure 2: VLBA image of Cygnus $A$ at $2 \mathrm{~cm}$. The synthesized beam is $(0.879 \times 0.52)$ mas $^{2}$. The image is contoured at $-1.5,1.5,3,6,12,24,48,96$ percent of the map peak at $0.453 \mathrm{Jy} / \mathrm{beam}$. The panels show a montage with selected $\mathrm{OH}$ absorption spectra. The bottom right panel shows the absorption spectrum integrated over the whole source.

\section{Results}

\subsection{The Effelsberg survey}

Of the originally selected 31 Seyfert 2 galaxies listed in Table 1, 27 sources were observed at $6 \mathrm{GHz}$ and 20 sources were observed at $4.7 \mathrm{GHz}$ with the 100-m Effelsberg telescope. We detected absorption at $6 \mathrm{GHz}$ towards five sources. The spectra of two of them, NGC 3079 and NGC 5793, are shown in Fig. 1. Absorption was detected towards the centre of each source, with wide velocity components and narrow troughs at the systemic velocities of the two lines. In NGC 3079 (Fig 1, left panel), the absorption width is $800 \mathrm{~km} / \mathrm{s}$, with two deeper components corresponding to the two transitions (6031 MHz and $6035 \mathrm{MHz}$ ) at the systemic velocity. The maximum line opacity is $\tau=0.05$. Two further lines corresponding to the two transitions were also observed blueshifted relative to the systemic velocity by $100 \mathrm{~km} / \mathrm{s}$, indicating the presence of some outflowing gas. Alternatively, these two troughs, symmetric around the 6035-MHz line, might suggest the presence of gas rotating around a central source. The VLBI jets in this source, a Seyfert 2 galaxy $16 \mathrm{Mpc}$ away, extend to $1.5 \mathrm{pc}$ from the central engine [14]; the 1.6-GHz absorption in this source has been found to come from nuclear region [15] and in addition to the broad velocity width, suggest that the 6-GHz absorption was also likely to come from the central region, where the torus is expected to be. No absorption was observed in the 4.7-GHz transition.

The absorption spectrum in NGC 5793 also shows a very broad line width, which ranges up to $1000 \mathrm{~km} / \mathrm{s}$ (Fig.1, right panel). Two narrow troughs, corresponding to the two transitions at the 
systemic velocity and corresponding to a maximum line opacity of $\tau \sim 0.034$, were observed. Both these sources show previously detected $\mathrm{OH}$ in absorption in the lower state transitions at $1.6 \mathrm{GHz}[16]$, [17].

\subsection{Cygnus A}

The 2-cm continuum image of Cygnus A (Fig. 2) shows a compact radio source, extending east-west up to $4 \mathrm{pc}$ from the core, with a peak flux density of $453 \mathrm{mJy} / \mathrm{beam}$. The noise in the map is $5 \mathrm{mJy} / \mathrm{beam}$. Fig. 2 shows the spectra for different positions at the source. We report a tentative detection of the rotationally-excited $\mathrm{OH}$ transition at $13.434 \mathrm{GHz}$ towards the centre of Cygnus A. The apparent optical depth derived from the ratios of intensities of peak absorption and adjacent continuum is $\tau=0.125$ with a line FWHM corresponding to $\sim 90 \mathrm{~km} / \mathrm{s}$. The absorption profile towards the lobes is suggestive that part of the gas is diffuse and is surrounding the inner jets, whereas a deeper and broader absorption profile is seen towards the core. The profile is strongest when integrated over the entire area containing continuum emission, and again seems to indicate that the gas is spread over the whole source, with prevalence towards the central region. Further tests are needed to confirm this result and to rule out a spurious feature due to low-level instrumental effects only detectable after long integration times.

\section{Conclusions}

We have detected rotationally-excited, broad $\mathrm{OH}$ lines in absorption towards five of the 27 sources observed with the 100-m telescope in Effelsberg. This yields a detection rate of $19 \%$, which is higher than the detection rates achieved in previous surveys. Those previous studies mainly targeted red quasars, where an infrared excess is indicative of large columns of dust towards the line of sight (e.g. [18], [19]). The source selection in our study was based on X-ray column densities. This is the first time this has been done. The observed line widths range from a few $100 \mathrm{~km} / \mathrm{s}$ to $2000 \mathrm{~km} / \mathrm{s}$, suggesting that the gas in all sources is close to the central region, either rotating around the central engine, or infalling/outflowing. We have found that the new 6-GHz detections have absorption in the ground state transition at $1.6 \mathrm{GHz}$. This does not support the hypothesis of radiative excitation models alone to explain the previous lack of molecular detections. However, given the high X-ray absorbing columns in our systems, the non-detections still need to be explained. Since the nature of the absorbing material is unknown, one possibility is that it may be non-molecular in most galaxies.

We have found that in the five systems with detections the lines are strong and were visible after short integration times, whereas in the non-detections no lines were visible even after longer integration times. This bimodal distribution of absorptions could be explained assuming the presence of compact clouds crossing the line of sight in a few sources.

We also report a tentative $\mathrm{OH}$ detection at $13.4 \mathrm{GHz}$ towards the powerful, nearby galaxy Cygnus A. This result needs further study to be confirmed. The compactness of the radio source at this frequency indicates that the absorbing material lies within $4 \mathrm{pc}$ along the line of sight to the central engine. This is consistent with most recent results based on high-resolution IR observations of NGC 1068, which show a torus size that is no more than a few parsecs, e.g. [20], [21]. 


\section{References}

[1] R. Antonucci, Unified models for active galactic nuclei and quasars, ARA\&A (1993) 31473

[2] P. Maloney; M. C. Begelman; M. J. Rees, Radiative excitation of molecules near powerful compact radio sources, ApJ (1994) 432606

[3] J.H. Krolik; M.C. Begelman, Molecular tori in Seyfert galaxies - Feeding the monster and hiding it, ApJ (1988) 32970

[4] J.T. Schmelz; W.A. Baan; A.D. Haschick et al., An Arecibo survey for extragalactic hydroxyl absorption. I - Presentation of results, AJ (1986) 921291

[5] W.A. Baan, Willem; A. Haschick et al. Hydroxyl in galaxies. I - Surveys with the NRAO 300 FT telescope, AJ (1992) $\mathbf{1 0 3} 728$

[6] L. Staveley-Smith; R.P. Norris; J.M. Chapman et al. A southern OH megamaser survey, MNRAS (1992) 258725

[7] R. Antonucci; T. Hurt; A. Kinney, Evidence for a Quasar in the Radio Galaxy Cygnus-A from Observation of Broadline Emission, Nature (1994) 371313

[8] S. Ueno; K. Koyama; M. Nishida et al. X-ray observations of Cygnus A using the GINGA satellite, ApJ (1994) 4311

[9] J.E. Conway; P.R. Blanco, HI Absorption Toward the Nucleus of the Powerful Radio Galaxy Cygnus A: Evidence for an Atomic Obscuring Torus?, ApJ (1995) 449131

[10] R. Barvainis; R. Antonucci, Search for CO absorption from a molecular torus in Cygnus A, AJ (1994) 1071291

[11] A. Fuente; J.H. Black; J. Martín-Pintado et al. Tentative Detection of CO+ toward Cygnus A, ApJ (2000) 545113

[12] J. Black, The Molecular Astrophysics of Stars and Galaxies (1997), 469

[13] U. Bach; T.P. Krichbaum; E. Middelberg et al. Spectral Properties of the Core and the VLBI-Jets of Cygnus A, evn.conf (2004) 155

[14] A.S. Trotter; L.J. Greenhill; J.M. Moran et al. Water Maser Emission and the Parsec-Scale Jet in NGC 3079, ApJ (1998) 495740

[15] Y., Hagiwara; H.R. KlÃúckner; W. Baan VLBI imaging of OH absorption: the puzzle of the nuclear region of NGC3079, MNRAS (2004) 3531055

[16] W.A. Baan; J.A. Irwin, The Nuclear Structure of NGC 3079, ApJ (1995) 446602

[17] Y. Hagiwara; P.J. Diamond; N. Nakai et al. Probing circumnuclear molecular gas in NGC 5793 with OH absorption, A\&A (2000) $\mathbf{3 6 0} 49$

[18] S.J. Curran; M.T. Whiting; M.T. Murphy et al. A survey for redshifted molecular and atomic absorption lines - I. The Parkes half-Jansky flat-spectrum red quasar sample, MNRAS (2006) 371431

[19] M.J. Drinkwater; F. Combes; T. Wiklind, A search for molecular absorption in the tori of active galactic nuclei, A\&A (1996) 312771

[20] W. Jaffe; K. Meisenheimer; H. Röttgering et al, The central dusty torus in the active nucleus of NGC 1068, nature (2004) 02531

[21] A. Poncelet; G. Perrin; H. Sol A new analysis of the nucleus of NGC 1068 with MIDI observations, $A \& A$ (2006) 450483 